\title{
Quantitative Measurements on the Human Ascending Aortic Flow Using 2D Cine Phase-Contrast Magnetic Resonance Imaging*
}

\author{
Suguru YOKOSAWA**, Masanori NAKAMURA**, Shigeo WADA**, \\ Haruo ISODA***, Hiroyasu TAKEDA*** and Takami YAMAGUCHI ${ }^{* *}$
}

The flow in the human ascending aorta was quantified using two-dimensional (2D) cine phase-contrast magnetic resonance imaging (MRI). The quality and reliability of the method were demonstrated with a specially designed phantom model; the flow rate determined with the MRI agreed well with that obtained with a measuring cylinder. The method was then used to measure the aortic blood flow of three healthy human volunteers. The velocity profiles at the supra-aortic valvular plane and ascending aortic plane (approximately 2 and $5 \mathrm{~cm}$ distal to the aortic valve, respectively) were significantly different. At the peak of systole, the profile was almost axisymmetric at the supra-aortic valvular plane, while it was skewed towards the anterior side of the vessel at the ascending aorta. The Reynolds number, volume flow rate, and stroke volume were all within the normal physiological range. This study demonstrated that the 2D cine phase-contrast MRI technique can be used to provide detailed information on the flow velocity and configuration of a blood vessel, making it a promising tool for analyzing complex hemodynamics in the aorta.

Key Words: Measurement, Biomechanics, Image Processing, Cine Phase Contrast Magnetic Resonance Imaging, Blood Flow, Aorta, Velocity Profile

\section{Introduction}

The aorta is the main trunk of the vertebrate circulation system. Its root is directly connected to the left ventricle of the heart. Due to the complex configuration and function of the left ventricle ${ }^{(1)}$, aortic blood flow has a complex three-dimensional nature ${ }^{(2)}$.

There have been several attempts to quantify aortic flow dynamics. Using a constant temperature hot-film anemometer, Paulsen et al $^{(3)}$ showed that the velocity profile in the ascending aorta matched that of a flat transitional flow with a slight skew towards the posterior and left wall of the aorta. By contrast, studies performed by Rossvoll et al. ${ }^{(4)}$ showed an anteriorly skewed velocity

* Received 15th June, 2005 (No. 05-4078)

** Department of Bioengineering and Robotics, Graduate School of Engineering, Tohoku University, Aoba 6-6-01, Sendai 980-8579, Japan.

E-mail: shigeo@pfsl.mech.tohoku.ac.jp

*** Department of Radiology, Hamamatsu University School of Medicine, Handayama 1-20-1, Hamamatsu 431-3192, Japan profile in the aortic annulus. Non-uniformity of the velocity profile at various levels of the ascending aorta ${ }^{(5)}$, as well as in the left ventricular outflow $\operatorname{tract}^{(6)-(9)}$, has also been reported. As exemplified above, general agreement has not been reached on the spatial distribution of aortic flow, and the direction of the skew in particular, due probably to the limited resolution of available measurements both in time and space. However, all researchers are in agreement that the velocity profile in the aortic annulus is not uniform.

Recent developments in cine phase-contrast magnetic resonance imaging (MRI) permit more quantitative measurements of the flow velocity in the heart ${ }^{(1),(10)}$ and blood vessels $^{(11)-(13)}$. The technique is non-invasive and applicable to most of the blood vessels in a human body, particularly to vessels in the interior of the skull and the pelvic region, which are not accessible to ultrasound examinations. Furthermore, the simultaneous acquisition of velocity and geometric data of a blood vessel makes it possible to estimate the blood flow rate, which is of great advantage not only from an engineering viewpoint, but also for clinical requirements. 
This study analyzed the unsteady flow velocity profile at the supra-aortic valvular plane and at the ascending aortic plane quantitatively using two-dimensional (2D) cine phase-contrast MRI. The reliability of MRI measurements was demonstrated in test experiments on a specially designed flow phantom model.

\section{Materials and Methods}

Two-dimensional cine phase-contrast MR examinations on a phantom model and human volunteers were carried out using a Signa Infinity Echo Speed 1.5 T MR system with the Excite option (GE, USA). The following MR parameters were used: repetition time (TR) $29 \mathrm{~ms}$ for the phantom model and $33 \mathrm{~ms}$ in vivo; echo time (TE) $5.75 \mathrm{~ms}$ for the phantom model and $6.3 \mathrm{~ms}$ in vivo; velocity encoding range $(\mathrm{VENC}) 150 \mathrm{~cm} / \mathrm{s}$; flip angle $30 \mathrm{de}-$ grees; slice thickness $5 \mathrm{~mm}$; matrix $192 \times 192$; and field of view (FOV) $32 \times 24 \mathrm{~cm}$ with a spatial resolution of $1.25 \times 1.25 \mathrm{~mm} /$ pixel. All images were acquired using an ECG-gated gradient echo sequence.

With 2D cine phase-contrast MRI, we could obtain a magnitude image that provided anatomical information, as well as phase-contrast images that provided velocity data in the three orthogonal directions. The acquired magnitude and phase-contrast images were transferred to a PC for the flow analysis. In phase-contrast images, the brightness is proportional to the magnitude of the velocity (the velocity can be either positive or negative depending on the direction of the flow projected onto the scanned plane). Here, flow heading from the paper to the reader is shaded white. The region of interest, which was the aortic lumen boundary for the human measurements, was traced manually on the magnitude image at each frame. The outline of the defined region was then copied and superimposed onto the corresponding phase-contrast image. Finally, the brightness of the pixels within the defined region was converted into the flow velocity. The flow rate was calculated from the axial velocities within the defined region at each moment in time, and the stroke volume was estimated by integrating the flow rate over the entire cycle.

Phantom model experiments were used to examine the reliability of the $2 \mathrm{D}$ cine phase-contrast MR measurements. Figure 1 illustrates the experimental setup with the phantom model, which consisted of a polyvinyl-chloride pipe (JIS K6742-506) with an inner lumen diameter of $2 \mathrm{~cm}$, a flow reservoir, and a pump. The test fluid was a $40 \%$ glycerol solution with a density of $1100 \mathrm{~kg} / \mathrm{m}^{3}$ and viscosity of $0.0042 \mathrm{~Pa} \cdot \mathrm{s}$. A 2.5 -m-long straight zone was prepared in proximity to the MRI. Note that the length of this straight zone was not sufficient to permit full development of Poiseuille flow; rather, it was designed to dampen the flow disturbances created by the pump. Steady flow with various flow rates was achieved using two different pumps (Nikkiso Magpon CP20PPRV-10 and CP40-PPRV10, Nikkiso, Japan), depending on the flow rate. The flow rate was controlled stepwise such that the cross-sectional average flow velocity was $0.3-0.7 \mathrm{~m} / \mathrm{s}$; the corresponding Reynolds numbers were 1570-3670. The flow rate was calibrated by measuring the volume of the outflow with a measuring cylinder at the end of the phantom model circuit during the experiment. In the actual experiment, two cylindrical bottles filled with $\mathrm{NiSO}_{4}$ solution were placed along both sides of the measurement section of the pipe to obtain a sufficient intensity of nuclear magnetic resonance signals released by excited protons.

For the human measurements, data were acquired from three male volunteers with normal electrocardiograms (ECGs) who had no history of cardiovascular disease. Detailed information about the subjects and the sites where the blood flow velocity profile was measured are shown in Table 1. Written informed consent was obtained

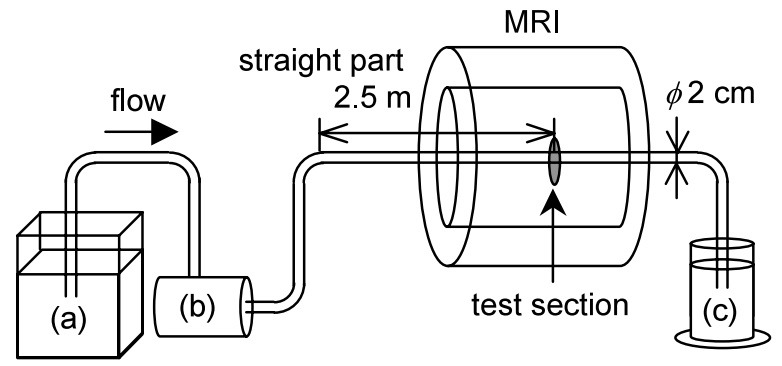

Fig. 1 MR flow phantom with the (a) reservoir, (b) pump, and (c) measuring cylinder

Table 1 General information about the subjects

\begin{tabular}{cccc}
\hline & subject 1 & subject 2 & subject 3 \\
\hline sex & male & male & male \\
age & 28 & 34 & 22 \\
heart rate $[\mathrm{bpm}]$ & 66 & 66 & 55 \\
height $[\mathrm{m}]$ & 1.80 & 1.73 & 1.72 \\
weight $[\mathrm{kg}]$ & 74 & 65 & 60 \\
measured site $[\mathrm{cm}]$ & 2 & 2 & 5 \\
\hline
\end{tabular}

"measured site" refers to the location of a transverse section scanned with the $2 \mathrm{D}$ cine phase-contrast

MRI defined as a distance from the aortic valve along the curvature of the aorta. 
from all volunteers with the approval of the Human Study Committee of Tohoku University. The subject was laid in the supine position on a bed equipped with the MR scanner. Then scout scans of the coronal, sagittal, and transverse planes were performed to identify the aortic valve and ascending aorta. The scout scan of the coronal plane for subject 1 is presented in Fig. 2. The slice to be scanned to actually measure the phase and magnitude images was then determined based on the scout scans so that it transversely covered a plane $2 \mathrm{~cm}$ (subject 1 and 2) or $5 \mathrm{~cm}$ (subject 3 ) distal to the aortic valve. A series of 20 equidistant images per heart beat interval were acquired for each subject using ECG synchronization. The trigger pulse was obtained from the R-wave of the ECG. The time interval between data acquisitions was $45 \mathrm{~ms}$ (subject 1 and 2) and $54 \mathrm{~ms}$ (subject 3). An example of the magnitude and

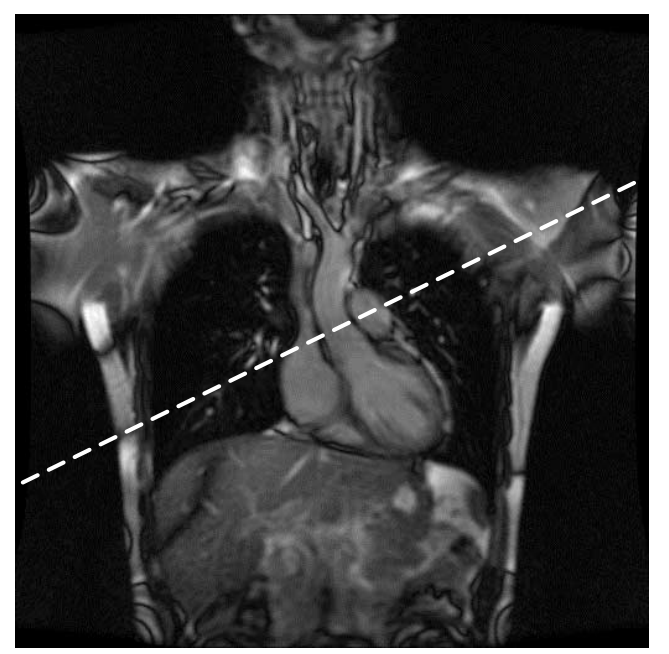

Fig. 2 A scout scan of the coronal plane of subject 1, used to identify the aortic valve and the ascending aorta. The dashed line in the figure indicates the scanned section used for the actual measurements

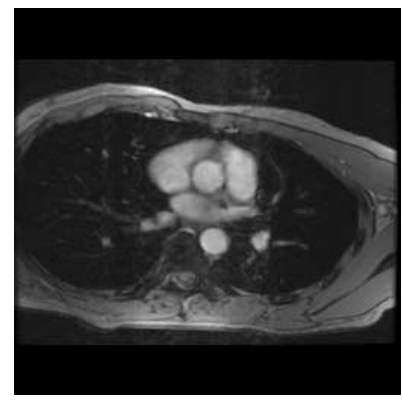

(a)

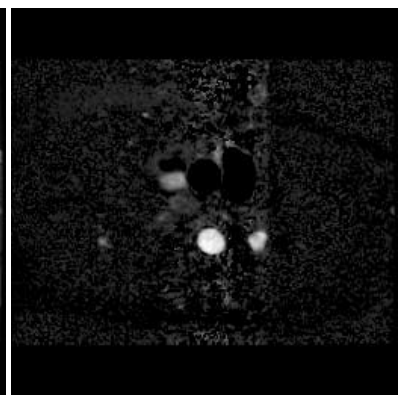

(b)
Fig. 3 An example of the magnitude (left) and phase-contrast (right) images through the transverse plane $2 \mathrm{~cm}$ above the aortic valve for subject 1 . The images are viewed from the inferior side, with the anterior side located towards the top of the figure phase-contrast images for subject 1 is shown in Fig. 3.

\section{Results}

The 2D cine phase-contrast MR examinations were evaluated using the phantom model. Figure 4 shows the correlation between the volume flow rates calculated from the phase image of a transverse slice through the phantom model and those measured with the measuring cylinder. There was good agreement between the values, even at higher flow rates where some turbulence might be expected.

In the human study, the intra-observer variation for estimating the flow rate was assessed as a coefficient of variation $(\mathrm{SD} /$ mean $\times 100 \%)$ for five randomized trials of tracing the aortic lumen on subject 3 by the same ob-

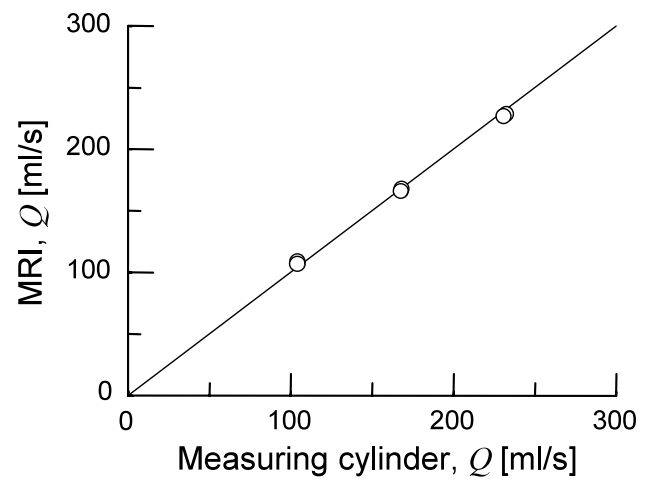

Fig. 4 Correlation curve between the flow rate determined with the 2D cine phase-contrast MRI and the flow value obtained with the measuring cylinder

Table 2 Coefficient of variation for the area, average velocity, and flow rate over five trials of tracing the region of interest in subject 3

\begin{tabular}{rccc}
\hline & \multicolumn{3}{c}{ coefficient of variation [\%] } \\
time [ms] & area & velocity & flow rate \\
\hline 1 & 2.8 & 1.9 & 1.0 \\
55 & 1.3 & 1.4 & 0.5 \\
110 & 2.2 & 2.7 & 0.8 \\
164 & 3.1 & 2.9 & 0.6 \\
219 & 2.4 & 2.0 & 0.8 \\
273 & 1.8 & 1.2 & 0.7 \\
328 & 2.8 & 1.4 & 1.8 \\
382 & 1.5 & 1.2 & 2.3 \\
437 & 4.4 & 1.8 & 2.7 \\
491 & 1.1 & 0.8 & 1.5 \\
546 & 1.8 & 0.8 & 1.0 \\
600 & 2.5 & 1.3 & 1.0 \\
655 & 2.3 & 1.0 & 1.4 \\
710 & 1.3 & 1.3 & 0.9 \\
764 & 1.8 & 4.2 & 2.6 \\
819 & 2.0 & 2.1 & 3.7 \\
873 & 2.4 & 1.5 & 2.6 \\
928 & 2.8 & 5.0 & 7.1 \\
982 & 1.8 & 45.7 & 44.7 \\
1037 & 3.5 & 5.2 & 4.7 \\
\hline & & & \\
& & &
\end{tabular}



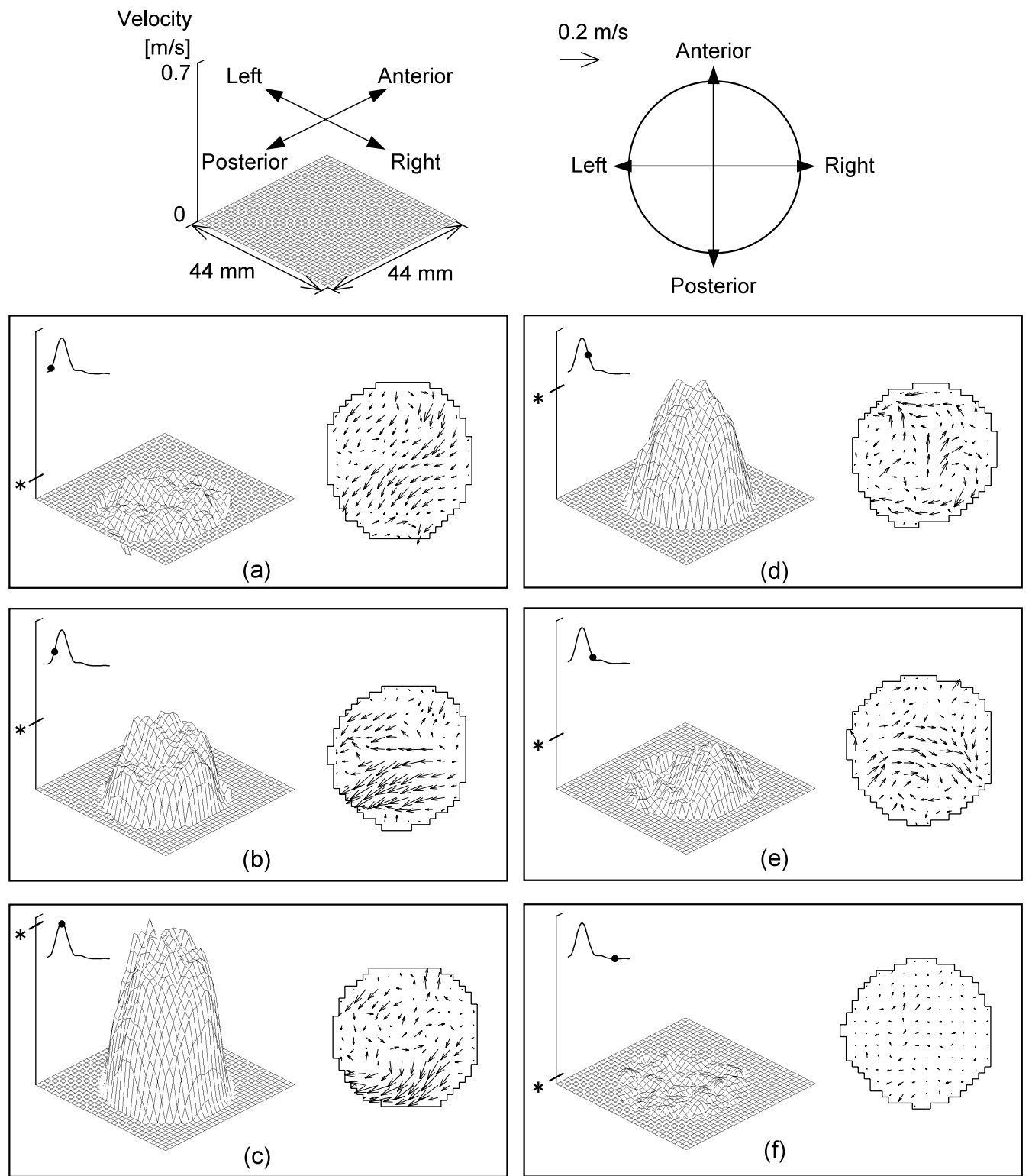

Fig. 5 Contour plots of the axial velocity and vector plots of the secondary flows at the plane $2 \mathrm{~cm}$ distal to the aortic valve for subject 1 : (a) 1, (b) 91 , (c) 182 , (d) 273 , (e) 364 , and (f) $682 \mathrm{~ms}$, as indicated by the flow rate curve on each contour plot. The orientation of the view for each plot is shown at the top, and “*” indicates the maximum velocity for each time

server. Table 2 summarizes the values of the coefficient of variation calculated for the area, spatially averaged velocity, and flow rate at all measured time points. All the coefficients of variation, except the velocity and flow rate at $t=982 \mathrm{~ms}$, were less than $5 \%$, which was sufficiently small to demonstrate the reproducibility of our 2D cine phase-contrast MR velocity measurement technique. The probable cause for the large coefficient of variation at $t=982 \mathrm{~ms}$ was an extremely low value of the flow velocity at that time in diastole.

Figures 5 and 6 show contour plots of the axial velocity and vector plots of the secondary flows at the plane $2 \mathrm{~cm}$ distal to the aortic valve at $t=1,91,182,273,364$ and $682 \mathrm{~ms}$ for subjects 1 and 2 . For convenience, hereafter this plane is referred to as the supra-aortic valvular plane. The view angle is indicated at the top of the figures, and a positive flow velocity indicates that the flow travels upward to the head. The axial velocity profile at the aortic annulus showed a similar trend between the subjects during the cardiac cycle. In early systole, the flow velocity was relatively high near the anterior side of the vessel (Figs. 5 (b) and 6(b)). As systole progressed, the velocity at the central part increased, resulting in an approximately axisymmetric velocity profile at mid-systole (Figs. 5 (c) and $6(\mathrm{c})$ ). Between middle and late systole, the velocity profile became slightly skewed, with faster flow devel- 

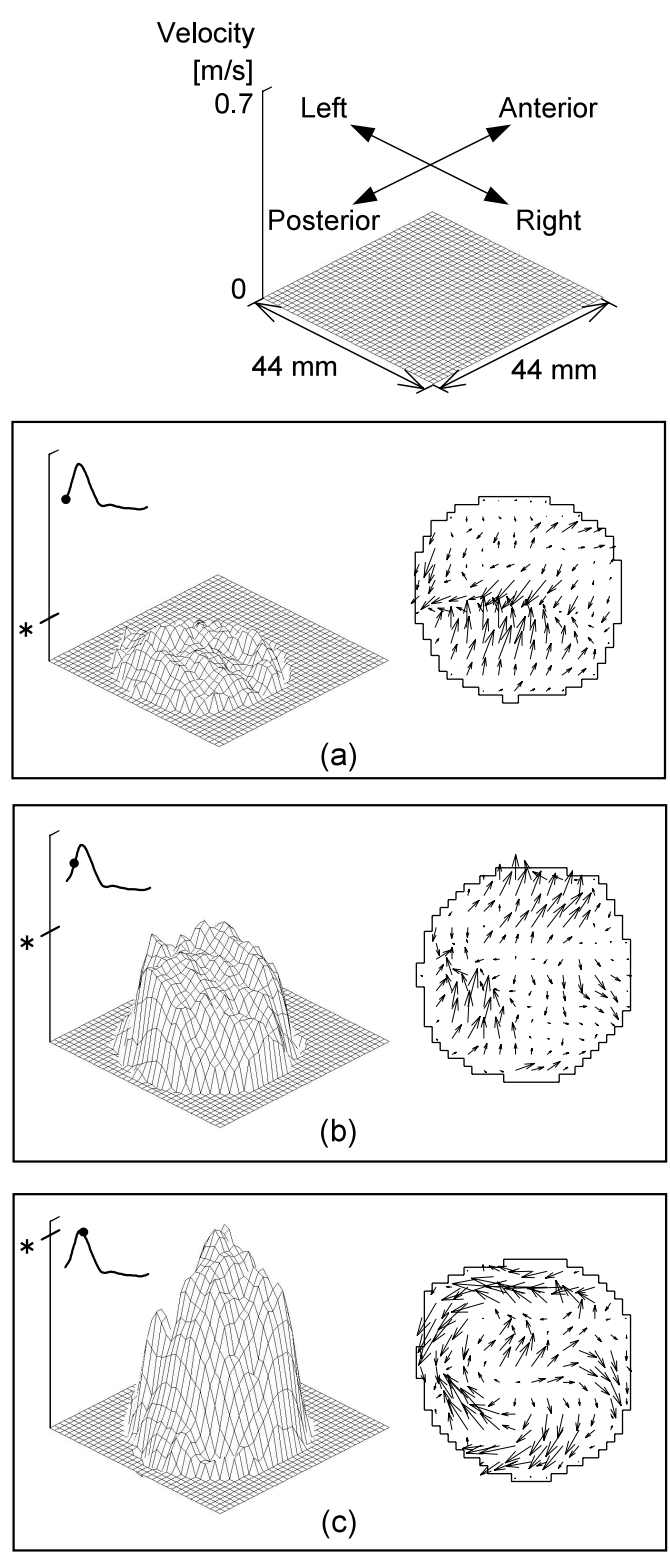
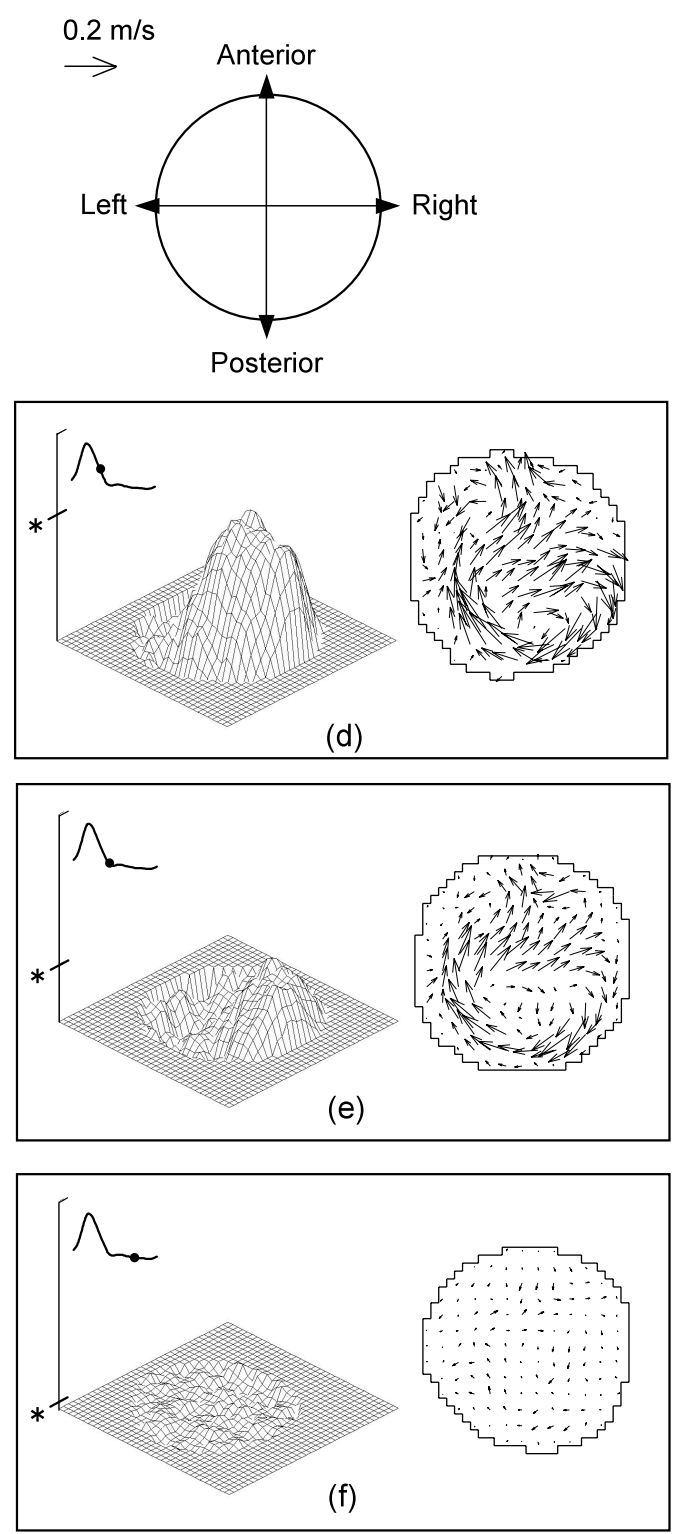

(f)

Fig. 6 Contour plots of the axial velocity and vector plots of the secondary flows at the plane $2 \mathrm{~cm}$ distal to the aortic valve for subject 2: (a) 1, (b) 91 , (c) 182 , (d) 273 , (e) 364 , and (f) $682 \mathrm{~ms}$, as indicated by the flow rate curve on each contour plot. The orientation of the view for each plot is shown at the top, and "*" indicates the maximum velocity for each time

oping towards the right side of the vessel (Figs. 5 (d) and 6 (d)). In late systole, a regurgitating flow was identified at the left side of the vessel. During diastole, the flow velocity was almost zero and contained only small fluctuations. By contrast, the secondary flows varied widely between the subjects. In subject 1 , the secondary flows were globally directed towards the left posterior side of the vessel in early systole (Fig. 5(b)). After the peak of systole, the secondary flows were randomly oriented (Fig. 5 (d)). The secondary flows were stronger in subject 2 . In this subject, some clockwise swirls were observed near the posterior wall (Fig. 6 (d) and (e)).

Figure 7 illustrates contour plots of the axial velocity and vector plots of the secondary flow at the plane $5 \mathrm{~cm}$ distal to the aortic valve at $t=1,55,164,273,382$, and $819 \mathrm{~ms}$ for subject 3 . Hereafter, this plane is referred to the ascending aortic plane. In early systole, the velocity profile was almost flat. As systole progressed, the flow velocity increased at the right side of the vessel. Then, the position of the higher flow velocity moved counterclockwise towards the anterior side of the vessel between middle and late systole. At the same time, backflow occurred at the left posterior side of the aorta, that is, the opposite side of the higher flow velocity. The secondary flow showed a counterclockwise rotational pattern consistent with the movement of the higher axial velocity fluid. 

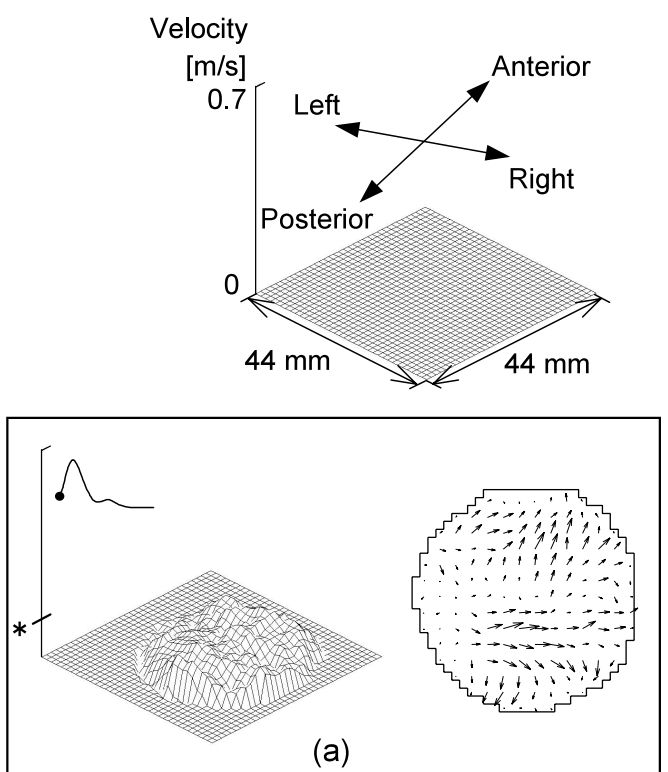

(a)
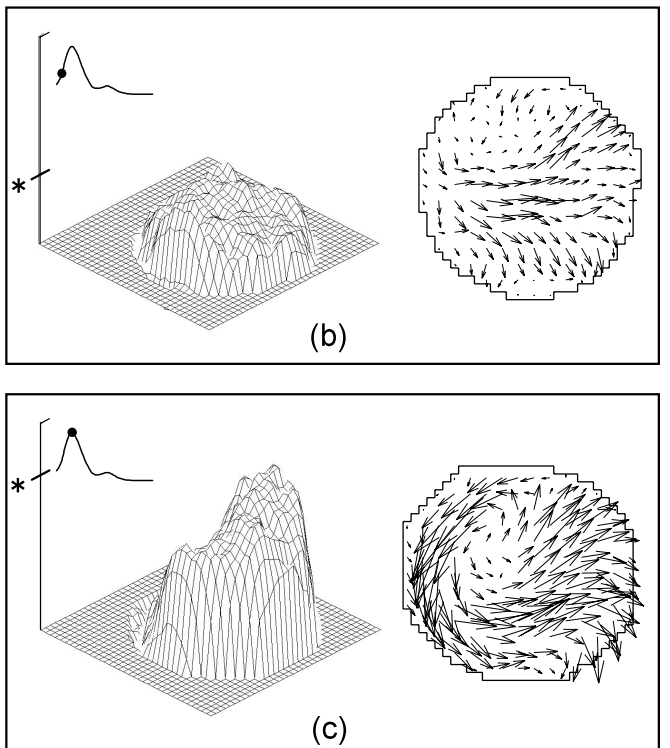
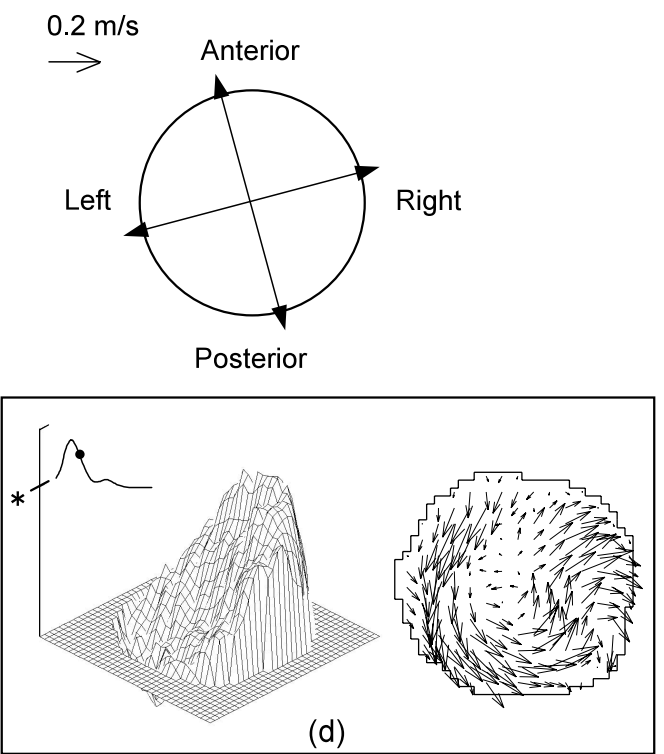

(d)
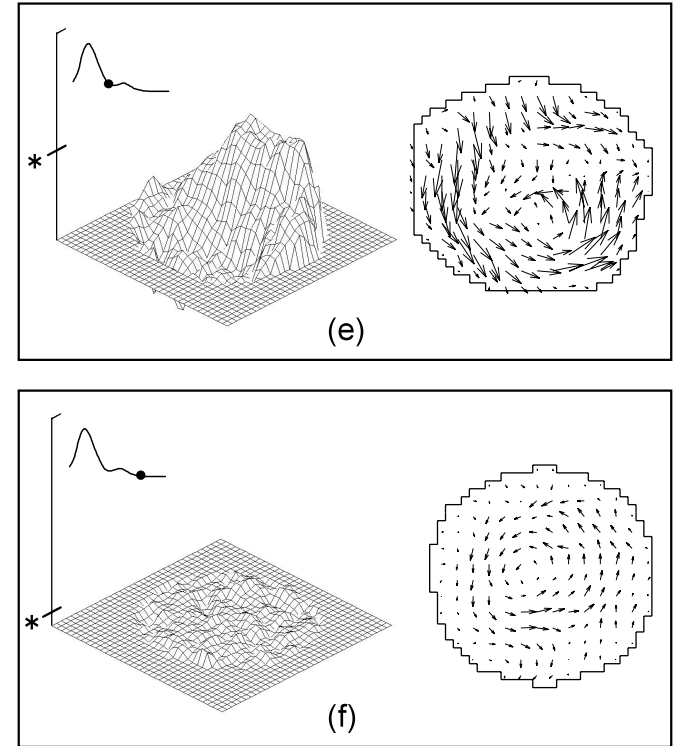

Fig. 7 Contour plots of the axial velocity and vector plots of the secondary flows at the plane $5 \mathrm{~cm}$ distal to the aortic valve for subject 3: (a) 1, (b) 55 , (c) 164 , (d) 273 , (e) 382 , and (f) $819 \mathrm{~ms}$, as indicated by the flow rate curve on each contour plot. The orientation of the view for each plot is shown at the top, and "*" indicates the maximum velocity for each time. Note that the orientation of this figure has shifted obliquely compared to Figs. 5 and 6

The diastolic flow at the ascending aortic plane was similar to that at the supra-valvular plane, i.e., the flow velocity was almost zero.

The respective spatially-averaged, maximum, and minimum axial velocities during the cardiac cycle were $0.37,0.60$, and $-0.50 \mathrm{~m} / \mathrm{s}$ for subject $1,0.24,0.65$, and $-0.16 \mathrm{~m} / \mathrm{s}$ for subject 2 , and $0.21,0.60$, and $-0.13 \mathrm{~m} / \mathrm{s}$ for subject 3 . The Reynolds numbers calculated using the spatially averaged axial velocity at the peak of systole and the diameter of the vessel lumen were 2620 (subject 1), 2360 (subject 2), and 2310 (subject 3).

The flow rate calculated by integrating the flow veloc-

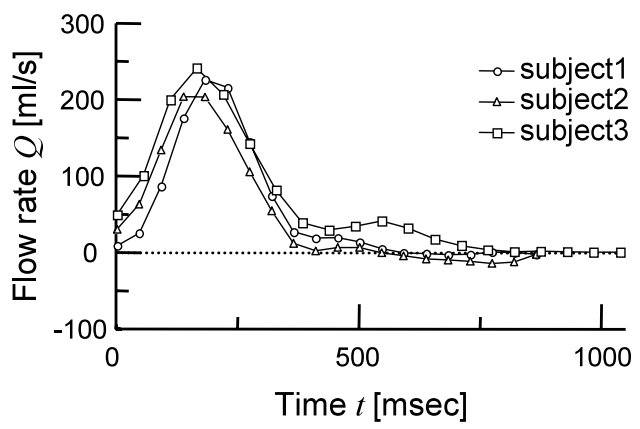

Fig. 8 Time history of the aortic flow rate measured using 2D cine phase-contrast MRI 
ity over the section area is plotted against time in Fig. 8. Here, the abscissa shows the elapsed time from the onset of systole. The calculated flow rate increased from the beginning of systole, reached a peak during mid systole, and decreased towards the end of systole. During diastole, the flow rate was almost zero. The stroke volume was 47.9, 42.6 , and $68.4 \mathrm{ml}$ for subjects 1,2 , and 3, respectively.

\section{Discussion}

The principles of cine phase contrast MR measurements have been verified by many pioneering studies $^{(14)-(18)}$. Given that, this study successfully evaluated the reliability of flow data acquired with our MR system using a phantom model and human subjects. The phantom model experiment demonstrated the accuracy of the flow rate data within the normal physiological range. The procedure tested with the phantom model measurements was then used for the human studies. The reproducibility of the flow rate estimations was confirmed by a statistical analysis of the intra-observer variation. The time history of the aortic flow rate concurred with the results of Jin et al. ${ }^{(19)}$ both quantitatively and qualitatively. The stroke volume estimated from the time history of the aortic flow rate was within the normal physiological range ${ }^{(20)}$. The calculated peak Reynolds number was also in a good agreement with physiological data ${ }^{(21)}$. These results suggest that our MR results are sufficiently reliable to discuss the hemodynamics in the aorta.

We observed that the position of the faster flow in the ascending aorta moved counterclockwise during the cardiac cycle. A comparison with flow data presented in Kilner et al. $^{(2)}$ suggests that the counterclockwise movement of the faster flow is associated with the development of helical flow in the aorta.

Previous studies ${ }^{(4)-(8)}$ have suggested that the velocity profile in the ventricular outflow tract is conserved to the aortic annulus and skewed to the ventricular septum. By contrast, we found that the velocity profile above the aortic valve was almost axisymmetric. This difference could be caused by the aortic valve between the aortic annulus and the supra-aortic valvular plane. In support of this hypothesis, De Hart et al. ${ }^{(22)}$ showed that blood flow is driven to the center of the vessel when passing through the aortic valve.

There was a difference in the velocity profiles at the supra-aortic valvular plane and the ascending aortic plane. This could have been induced by the spatial distortion of the aorta. Once blood enters the aorta, the bends and twists of the aorta increasingly affect it. Consequently, aortic flow has a different velocity profile from the flow at the aortic annulus ${ }^{(23)}$. This study demonstrated that the blood flow velocity profile at the supra-valvular plane was not at all uniform during systole. According to Nakamura et al. ${ }^{(24)}$ and Yokosawa et al. ${ }^{(25)}$, the velocity profile of the aortic inflow greatly influences the wall shear stress in the region proximal to the aortic arch. These studies suggest that the assumption of a flat velocity profile at the inlet of the aorta, which has been used frequently in previous studies $^{(23),(26),(27)}$, should be reconsidered.

There are some specific limitations to our study. Aortic flow is somewhat turbulent ${ }^{(28)}$, and turbulent flow limits the accuracy of the measurements. This might introduce some errors in the velocity data, although all the data presented here were within the normal physiological range. Another limitation is the fixed nature of the scanned section over the entire cardiac cycle. According to Jin et $a l .{ }^{(19)}$, the ascending aorta, including the aortic root, moves up and down over approximately $1 \mathrm{~cm}$ during the cardiac cycle. Therefore, the velocity profile presented here was not always measured at the "same" section from an anatomical point of view. Motion compensation strategies, such as section tracking ${ }^{(29)}$, which are now often used to reduce respiratory artifacts, might be adopted in the future to perform prospective motion corrections for the aorta.

\section{Conclusion}

The velocity profile of aortic flow was quantified using 2D cine phase-contrast MRI. The quality and reliability of this technique were demonstrated with phantom model measurements and human studies. The velocity profiles at the supra-aortic valvular plane and the ascending aortic plane were not uniform over the section and exhibited complex secondary flows. The Reynolds number, volume flow rate, and stroke volume computed from the measured data were all within the normal physiological range. In conclusion, the $2 \mathrm{D}$ cine phase-contrast MRI technique is capable of providing detailed information about the flow velocity and configuration of a blood vessel, making it a promising tool for analyzing complex hemodynamics in the aorta.

\section{Acknowledgement}

This work was supported by Research Fellowships of the Japan Society for the Promotion of Science for Young Scientists No. 06787 and Grant-in-Aid for Scientific Research No. 15086204, No. 17300138 and No. 16200031.

\section{References}

( 1 ) Kilner, P.J., Yang, G.Z., Mohiaddin, R.H., Firmin, D.N. and Longmore, D.B., Helical and Retrograde Secondary Flow Patterns in the Aortic Arch Studied by Three-Directional Magnetic Resonance Velocity Mapping, Circulation, Vol.88, No.5 Pt 1 (1993), pp.22352247.

( 2 ) Kilner, P.J., Yang, G.Z., Wilkes, A.J., Mohiaddin, R.H., Firmin, D.N. and Yacoub, M.H., Asymmetric Redirection of Flow through the Heart, Nature, Vol.404, No.6779 (2000), pp.759-761. 
( 3 ) Paulsen, P.K. and Hasenkam, J.M., Three-Dimensional Visualization of Velocity Profiles in the Ascending Aorta in Dogs, Measured with a Hot-Film Anemometer, J. Biomech., Vol.16, No.3 (1983), pp.201-210.

( 4 ) Rossvoll, O., Samstad, S., Torp, H.G., Linker, D.T., Skjaerpe, T., Angelsen, B.A. and Hatle, L., The Velocity Distribution in the Aortic Anulus in Normal Subjects: A Quantitative Analysis of Two-Dimensional Doppler Flow Maps, J. Am. Soc. Echocardiogr., Vol.4, No.4 (1991), pp.367-378.

( 5 ) Haugen, B.O., Berg, S., Brecke, K.M., Torp, H., Slordahl, S.A., Skaerpe, T. and Samstad, S.O., Blood Flow Velocity Profiles in the Aortic Annulus: A 3Dimensional Freehand Color Flow Doppler Imaging Study, J. Am. Soc. Echocardiogr., Vol.15, No.4 (2002), pp.328-333.

( 6 ) Kupari, M. and Koskinen, P., Systolic Flow Velocity Profile in the Left Ventricular Outflow Tract in Persons Free of Heart Disease, Am. J. Cardiol., Vol.72, No.15 (1993), pp.1172-1178.

( 7 ) Kupari, M., Hekali, P. and Poutanen, V.P., Cross Sectional Profiles of Systolic Flow Velocities in Left Ventricular Outflow Tract of Normal Subjects, Br. Heart. J., Vol.74, No.1 (1995), pp.34-39.

( 8 ) Zhou, Y.Q., Faerestrand, S., Matre, K. and Birkeland, S., Velocity Distributions in the Left Ventricular Outflow Tract and the Aortic Annulus Measured with Doppler Colour Flow Mapping in Normal Subjects, Eur. Heart J., Vol.14, No.9 (1993), pp.1179-1188.

( 9 ) Zhou, Y.Q., Faerestrand, S., Birkeland, S., Matre, K., Koller, M.E. and Husby, P., The Velocity Distribution in the Aortic Annulus at Different Times during Systole Is Mainly Determined by the Pattern of Flow Convergence in the Left Ventricular Outflow Tract - An Experimental Study Using Doppler Colour Flow Mapping, Clin. Physiol., Vol.15, No.6 (1995), pp.597-610.

(10) Ebbers, T., Wigstrom, L., Bolger, A.F., Wranne, B. and Karlsson, M., Noninvasive Measurement of TimeVarying Three-Dimensional Relative Pressure Fields within the Human Heart, ASME J. Biomech. Eng., Vol.124, No.3 (2002), pp.288-293.

(11) Kvitting, J.P., Ebbers, T., Wigstrom, L., Engvall, J., Olin, C.L. and Bolger, A.F., Flow Patterns in the Aortic Root and the Aorta Studied with TimeResolved, 3-Dimensional, Phase-Contrast Magnetic Resonance Imaging: Implications for Aortic ValveSparing Surgery, J. Thorac. Cardiovasc. Surg., Vol.127, No.6 (2004), pp.1602-1607.

(12) Markl, M., Draney, M.T., Hope, M.D., Levin, J.M., Chan, F.P., Alley, M.T., Pelc, N.J. and Herfkens, R.J., Time-Resolved 3-Dimensional Velocity Mapping in the Thoracic Aorta: Visualization of 3Directional Blood Flow Patterns in Healthy Volunteers and Patients, J. Comput. Assist. Tomogr., Vol.28, No.4 (2004), pp.459-468.

(13) Sakuma, H. and Higgins, C.B., Magnetic Resonance Measurement of Coronary Blood Flow, Acta. Paediatr. Suppl., Vol.93, No.446 (2004), pp.80-85.

(14) Klipstein, R.H., Firmin, D.N., Underwood, S.R., Rees, R.S. and Longmore, D.B., Blood Flow Patterns in the
Human Aorta Studied by Magnetic Resonance, Br. Heart J., Vol.58, No.4 (1987), pp.316-323.

(15) Meier, D., Maier, S. and Bosiger, P., Quantitative Flow Measurements on Phantoms and on Blood Vessels with MR, Magn. Reson. Med., Vol.8, No.1 (1988), pp.2534.

(16) Masuda, Y., Inoue, T., Imai, H. and Watanabe, S., Flow Measurement by MRI; Application of Phase Contrast Method, J. Jpn. Soc. Biorheol., (in Japanese), Vol.7, No.4 (1993), pp.107-110.

(17) Graves, M.J., Magnetic Resonance Angiography, Br. J. Radiol., Vol.70 (1997), pp.6-28.

(18) Lee, V.S., Spritzer, C.E., Carroll, B.A., Pool, L.G., Bernstein, M.A., Heinle, S.K. and MacFall, J.R., Flow Quantification Using Fast Cine Phase-Contrast MR Imaging, Conventional Cine Phase-Contrast MR Imaging, and Doppler Sonography: In Vitro and in Vivo Validation, AJR Am. J. Roentgenol., Vol.169, No.4 (1997), pp.1125-1131.

(19) Jin, S., Oshinski, J. and Giddens, D.P., Effects of Wall Motion and Compliance on Flow Patterns in the Ascending Aorta, ASME J. Biomech. Eng., Vol.125, No.3 (2003), pp.347-354.

(20) Lorenz, C.H., Walker, E.S., Morgan, V.L., Klein, S.S. and Graham, T.P. Jr., Normal Human Right and Left Ventricular Mass, Systolic Function, and Gender Differences by Cine Magnetic Resonance Imaging, J. Cardiovasc. Magn. Reson., Vol.1, No.1 (1999), pp.7-21.

(21) Caro, C.G., Pedley, T.J., Schroter, R.C. and Seed, W.A., The Mechanics of Circulation, (1978), pp.151-180, Oxford University Press, Oxford.

(22) De Hart, J., Peters, G.W., Schreurs, P.J. and Baaijens, F.P., A Three-Dimensional Computational Analysis of Fluid-Structure Interaction in the Aortic Valve, J. Biomech., Vol.36, No.1 (2003), pp.103-112.

(23) Shahcheraghi, N., Dwyer, H.A., Cheer, A.Y., Barakat, A.I. and Rutaganira, T., Unsteady and ThreeDimensional Simulation of Blood Flow in the Human Aortic Arch, ASME J. Biomech. Eng., Vol.124, No.4 (2002), pp.378-387.

(24) Nakamura, M., Wada, S. and Yamaguchi, T., Computational Analysis of Blood Flow in an Integrated Model of the Left Ventricle and the Aorta, Submitted to ASME Journal of Biomechanical Engineering.

(25) Yokosawa, S., Wada, S., Nakamura, M., Tsubota, K., Yamaguchi, T. and Isoda, H., Phase Contrast MRI Measurements and CFD Analysis of Hemodynamics in the Aorta, Proceedings of 2005 Summer Bioengineering Conference, 2 Pages (in CD), 2005 Summer Bioengineering Conference, Vail, CO, USA.

(26) Fujioka, H. and Tanishita, K., Computational Fluid Mechanics of the Blood Flow in an Aortic Vessel with Realistic Geometry, Clinical Application of Computational Mechanics to the Cardiovascular System, Edited by Yamaguchi, T., (2000), pp.99-117, Springer-Verlag, Tokyo.

(27) Mori, D. and Yamaguchi, T., Computational Fluid Dynamics Analysis of the Blood Flow in the Thoracic Aorta on the Development of Aneurysm, J. Jpn. Coll. Angiol., Vol.43 (2003), pp.94-97. 
(28) Yamaguchi, T., Kikkawa, S., Yoshikawa, T., Tanishita, K. and Sugawara, M., Measurement of Turbulence Intensity in the Center of the Canine Ascending Aorta with a Hot-Film Anemometer, J. Biomech. Eng., Vol.105, No.2 (1983), pp.177-187.
(29) McConnell, M.V., Khasgiwala, V.C., Savord, B.J., Chen, M.H., Chuang, M.L., Edelman, R.R. and Manning, W.J., Prospective Adaptive Navigator Correction for Breath-Hold MR Coronary Angiography, Magn. Reson. Med., Vol.37, No.1 (1997), pp.148-152. 